Bull. Korean Math. Soc. 49 (2012), No. 4, pp. 775-785

http://dx.doi.org/10.4134/BKMS.2012.49.4.775

\title{
A NOTE ON OSTROWSKI TYPE INEQUALITIES RELATED TO SOME $s$-CONVEX FUNCTIONS IN THE SECOND SENSE
}

\author{
ZHENG LIU
}

\begin{abstract}
Some errors in literatures are pointed out and corrected. A generalization of Ostrowski type inequalities for functions whose derivatives in absolute value are $s$-convex in the second sense is established. Special cases are discussed.
\end{abstract}

\section{Introduction}

In [2], by using the Hölder inequality, Dragomir and Agarwal first proved the following trapezoid type inequality.

Theorem 1. Suppose $a, b \in I \subseteq \mathbb{R}$ with $a<b$ and $f: I^{0} \rightarrow \mathbb{R}$ is differentiable and let $p>1$. If $\left|f^{\prime}\right|^{\frac{p}{p-1}}$ is convex on $[a, b]$, then

(1)

$$
\left|\frac{f(a)+f(b)}{2}-\frac{1}{b-a} \int_{a}^{b} f(t) d t\right| \leq \frac{b-a}{2(p+1)^{\frac{1}{p}}}\left[\frac{\left|f^{\prime}(a)\right|^{\frac{p}{p-1}}+\left|f^{\prime}(b)\right|^{\frac{p}{p-1}}}{2}\right]^{\frac{p-1}{p}} .
$$

Remark 1. If we assume $\left|f^{\prime}(x)\right| \leq M$ for $x \in[a, b]$ in Theorem 1, then (1) may reduce to

$$
\left|\frac{f(a)+f(b)}{2}-\frac{1}{b-a} \int_{a}^{b} f(t) d t\right| \leq \frac{M(b-a)}{2(p+1)^{\frac{1}{p}}} .
$$

In [6], Pearce and Pečarić using the Hölder inequality in a different way, but did not use the power-mean inequality as stated in the proof of Theorem 1 of [6], provided an improvement of the above result as follows.

Theorem 2. Suppose $a, b \in I \subseteq \mathbb{R}$ with $a<b$ and $f: I^{0} \rightarrow \mathbb{R}$ is differentiable. If the function $\left|f^{\prime}\right|^{q}(q \geq 1)$ is convex on $[a, b]$, then

$$
\left|\frac{f(a)+f(b)}{2}-\frac{1}{b-a} \int_{a}^{b} f(t) d t\right| \leq \frac{b-a}{4}\left[\frac{\left|f^{\prime}(a)\right|^{q}+\left|f^{\prime}(b)\right|^{q}}{2}\right]^{\frac{1}{q}}
$$

Received April 8, 2011.

2010 Mathematics Subject Classification. 26D15.

Key words and phrases. Ostrowski type inequality, convex function, s-convex function, Hölder inequality, averaged midpoint-trapezoid inequality. 
and

$$
\left|f\left(\frac{a+b}{2}\right)-\frac{1}{b-a} \int_{a}^{b} f(t) d t\right| \leq \frac{b-a}{4}\left[\frac{\left|f^{\prime}(a)\right|^{q}+\left|f^{\prime}(b)\right|^{q}}{2}\right]^{\frac{1}{q}} .
$$

Remark 2. If we assume $\left|f^{\prime}(x)\right| \leq M$ for $x \in[a, b]$ in Theorem 2, then (3) and (4) may reduce to

$$
\left|\frac{f(a)+f(b)}{2}-\frac{1}{b-a} \int_{a}^{b} f(t) d t\right| \leq \frac{M(b-a)}{4}
$$

and

$$
\left|f\left(\frac{a+b}{2}\right)-\frac{1}{b-a} \int_{a}^{b} f(t) d t\right| \leq \frac{M(b-a)}{4} .
$$

We recall that Hudzik and Maligranda in [4] has defined a function $f$ : $[0, \infty) \rightarrow \mathbb{R}$ is said to be $s$-convex in the second sense if

$$
f(\lambda x+(1-\lambda) y) \leq \lambda^{s} f(x)+(1-\lambda)^{s} f(y)
$$

holds for all $x, y \in[0, \infty), \lambda \in[0,1]$ and for some fixed $s \in(0,1]$. The class of $s$-convex functions in the second sense is usually denoted with $K_{s}^{2}$. It can be easily seen that for $s=1, s$-convexity reduces to ordinary convexity of functions defined on $[0, \infty)$. It is proved in [4] that all functions from $K_{s}^{2}, s \in(0,1)$ are nonnegative. Naturally, we may say a function $f:[0, \infty) \rightarrow \mathbb{R}$ is $s$-concave in the second sense for some fixed $s \in(0,1]$ if $-f \in K_{s}^{2}$. Thus we can conclude that $s$-concave function is always nonpositive for any $s \in(0,1)$.

In a recent paper [1], Alomari et al. used Hölder inequality to obtain the following Ostrowski type inequalities for functions whose derivatives in absolute value are $s$-convex in the second sense.

Theorem 3. Let $f: I \subset[0, \infty) \rightarrow \mathbb{R}$ be a differentiable mapping on $I^{\circ}$ such that $f^{\prime} \in L[a, b]$, where $a, b \in I$ with $a<b$. If $\left|f^{\prime}\right|^{q}$ is s-convex in the second sense on $[a, b]$ for some fixed $s \in(0,1], p, q>1, \frac{1}{p}+\frac{1}{q}=1$ and $\left|f^{\prime}(x)\right| \leq M$, $x \in[a, b]$, then the following inequality holds:

$$
\left|f(x)-\frac{1}{b-a} \int_{a}^{b} f(t) d t\right| \leq \frac{M}{(1+p)^{\frac{1}{p}}}\left(\frac{2}{s+1}\right)^{\frac{1}{q}}\left[\frac{(x-a)^{2}+(b-x)^{2}}{b-a}\right]
$$

for each $x \in[a, b]$.

In the same paper [1], by using Hölder inequality in a different way, but did not use the power-mean inequality as stated in the proof of Theorem 4 of [1], the authors obtained another Ostrowski type inequalities for functions whose derivatives in absolute value are $s$-convex in the second sense as follows.

Theorem 4. Let $f: I \subset[0, \infty) \rightarrow \mathbb{R}$ be a differentiable mapping on $I^{\circ}$ such that $f^{\prime} \in L[a, b]$, where $a, b \in I$ with $a<b$. If $\left|f^{\prime}\right|^{q}$ is $s$-convex in the second 
sense on $[a, b]$ for some fixed $s \in(0,1]$ and $q \geq 1$, and $\left|f^{\prime}(x)\right| \leq M, x \in[a, b]$, then the following inequality holds:

$$
\left|f(x)-\frac{1}{b-a} \int_{a}^{b} f(t) d t\right| \leq M\left(\frac{2}{s+1}\right)^{\frac{1}{q}}\left[\frac{(x-a)^{2}+(b-x)^{2}}{2(b-a)}\right]
$$

for each $x \in[a, b]$.

It is clear that the inequality (8) is better than the inequality (7) since $(1+p)^{\frac{1}{p}}<2$ for any $p>1$. However, this does not mean the approach via the power mean inequality is a better approach than that through Hölder's inequality as stated in Remark 1 of [1], since the power-mean inequality has not been used in proving the inequality (8). It is just incorrectly relay an erroneous message from [6] as mentioned above. Moreover, it should be noticed that Theorem 5 in [1] and Theorem 4 in [5] are not valid since a nonnegative $\left|f^{\prime}\right|^{q}$ could not be a $s$-concave function for any fixed $s \in(0,1)$.

In this work, we will also use Hölder inequality in two different ways to provide generalizations of Ostrowski type inequalities for functions whose derivatives in absolute value are $s$-convex in the second sense. Some special cases with applications of the averaged midpoint-trapezoid inequalities in special means are discussed.

\section{Main results}

In order to prove our main theorems, we need the following lemma:

Lemma 1. Let $f: I \subset \mathbb{R} \rightarrow \mathbb{R}$ be a differentiable mapping on $I^{\circ}$ where $a, b \in I$ with $a<b$. If $f^{\prime} \in L([a, b])$, then for any $\lambda \in[0,1]$ and $x \in[a, b]$ the following equality holds:

$$
\begin{aligned}
& (1-\lambda) f(x)+\lambda \frac{f(a)+f(b)}{2}-\frac{1}{b-a} \int_{a}^{b} f(t) d t \\
= & \frac{(x-a)^{2}}{b-a} \int_{0}^{1}\left(t-\frac{\lambda}{2} \frac{b-a}{x-a}\right) f^{\prime}(t x+(1-t) a) d t \\
& -\frac{(b-x)^{2}}{b-a} \int_{0}^{1}\left(t-\frac{\lambda}{2} \frac{b-a}{b-x}\right) f^{\prime}(t x+(1-t) b) d t .
\end{aligned}
$$

Proof. From [3] we see that

$$
\begin{aligned}
& (1-\lambda) f(x)+\lambda \frac{f(a)+f(b)}{2}-\frac{1}{b-a} \int_{a}^{b} f(t) d t \\
= & \frac{1}{b-a}\left\{\int_{a}^{x}\left[t-\left(a+\lambda \frac{b-a}{2}\right)\right] f^{\prime}(t) d t+\int_{x}^{b}\left[t-\left(b-\lambda \frac{b-a}{2}\right)\right] f^{\prime}(t) d t\right\} .
\end{aligned}
$$

By changing the variable $t$ of the first integral to $a+(x-a) t$ and the variable $t$ of the second integral to $b-(b-x) t$ in $(10)$, we get $(9)$ at once. 
Theorem 5. Let the assumptions of Theorem 3 hold. Then for all $\lambda \in[0,1]$ and $x \in\left[a+\lambda \frac{b-a}{2}, b-\lambda \frac{b-a}{2}\right]$, the following inequality holds:

$$
\begin{aligned}
& ||(1-\lambda) f(x)+\lambda \frac{f(a)+f(b)}{2}-\frac{1}{b-a} \int_{a}^{b} f(t) d t \mid \\
& \leq \frac{M}{b-a}\left(\frac{1}{p+1}\right)^{\frac{1}{p}}\left(\frac{2}{s+1}\right)^{\frac{1}{q}}\left\{(x-a)^{2}\left[\left(\frac{\lambda}{2} \frac{b-a}{x-a}\right)^{p+1}+\left(1-\frac{\lambda}{2} \frac{b-a}{x-a}\right)^{p+1}\right]^{\frac{1}{p}}\right. \\
& \left.+(b-x)^{2}\left[\left(\frac{\lambda}{2} \frac{b-a}{b-x}\right)^{p+1}+\left(1-\frac{\lambda}{2} \frac{b-a}{b-x}\right)^{p+1}\right]^{\frac{1}{p}}\right\}
\end{aligned}
$$

Proof. By Lemma 1 and notice $x \in\left[a+\lambda \frac{b-a}{2}, b-\lambda \frac{b-a}{2}\right]$ implies that $0 \leq$ $\frac{\lambda}{2} \frac{b-a}{x-a} \leq 1$ and $0 \leq \frac{\lambda}{2} \frac{b-a}{b-x} \leq 1$, using the Hölder inequality, we have

$$
\begin{aligned}
& \left|(1-\lambda) f(x)+\lambda \frac{f(a)+f(b)}{2}-\frac{1}{b-a} \int_{a}^{b} f(t) d t\right| \\
& \leq \frac{(x-a)^{2}}{b-a} \int_{0}^{1}\left|t-\frac{\lambda}{2} \frac{b-a}{x-a} \| f^{\prime}(t x+(1-t) a)\right| d t \\
& +\frac{(b-x)^{2}}{b-a} \int_{0}^{1}\left|t-\frac{\lambda}{2} \frac{b-a}{b-x}\right|\left|f^{\prime}(t x+(1-t) b)\right| d t \\
& \leq \frac{(x-a)^{2}}{b-a}\left(\int_{0}^{1}\left|t-\frac{\lambda}{2} \frac{b-a}{x-a}\right|^{p} d t\right)^{\frac{1}{p}}\left(\int_{0}^{1}\left|f^{\prime}(t x+(1-t) a)\right|^{q} d t\right)^{\frac{1}{q}} \\
& +\frac{(b-x)^{2}}{b-a}\left(\int_{0}^{1}\left|t-\frac{\lambda}{2} \frac{b-a}{b-x}\right|^{p} d t\right)^{\frac{1}{p}}\left(\int_{0}^{1}\left|f^{\prime}(t x+(1-t) b)\right|^{q} d t\right)^{\frac{1}{q}} \\
& \leq \frac{(x-a)^{2}}{b-a}\left(\frac{1}{p+1}\right)^{\frac{1}{p}}\left(\frac{1}{s+1}\right)^{\frac{1}{q}}\left[\left(\frac{\lambda}{2} \frac{b-a}{x-a}\right)^{p+1}+\left(1-\frac{\lambda}{2} \frac{b-a}{x-a}\right)^{p+1}\right]^{\frac{1}{p}} \\
& \times\left[\left|f^{\prime}(x)\right|^{q}+\left|f^{\prime}(a)\right|^{q}\right]^{\frac{1}{q}} \\
& +\frac{(b-x)^{2}}{b-a}\left(\frac{1}{p+1}\right)^{\frac{1}{p}}\left(\frac{1}{s+1}\right)^{\frac{1}{q}}\left[\left(\frac{\lambda}{2} \frac{b-a}{b-x}\right)^{p+1}+\left(1-\frac{\lambda}{2} \frac{b-a}{b-x}\right)^{p+1}\right]^{\frac{1}{p}} \\
& \times\left[\left|f^{\prime}(x)\right|^{q}+\left|f^{\prime}(b)\right|^{q}\right]^{\frac{1}{q}} \\
& \leq \frac{M(x-a)^{2}}{b-a}\left(\frac{1}{p+1}\right)^{\frac{1}{p}}\left(\frac{2}{s+1}\right)^{\frac{1}{q}}\left[\left(\frac{\lambda}{2} \frac{b-a}{x-a}\right)^{p+1}+\left(1-\frac{\lambda}{2} \frac{b-a}{x-a}\right)^{p+1}\right]^{\frac{1}{p}} \\
& +\frac{M(b-x)^{2}}{b-a}\left(\frac{1}{p+1}\right)^{\frac{1}{p}}\left(\frac{2}{s+1}\right)^{\frac{1}{q}}\left[\left(\frac{\lambda}{2} \frac{b-a}{b-x}\right)^{p+1}+\left(1-\frac{\lambda}{2} \frac{b-a}{b-x}\right)^{p+1}\right]^{\frac{1}{p}} \\
& =\frac{M}{b-a}\left(\frac{1}{p+1}\right)^{\frac{1}{p}}\left(\frac{2}{s+1}\right)^{\frac{1}{q}}\left\{(x-a)^{2}\left[\left(\frac{\lambda}{2} \frac{b-a}{x-a}\right)^{p+1}+\left(1-\frac{\lambda}{2} \frac{b-a}{x-a}\right)^{p+1}\right]^{\frac{1}{p}}\right.
\end{aligned}
$$




$$
\left.+(b-x)^{2}\left[\left(\frac{\lambda}{2} \frac{b-a}{b-x}\right)^{p+1}+\left(1-\frac{\lambda}{2} \frac{b-a}{b-x}\right)^{p+1}\right]^{\frac{1}{p}}\right\},
$$

since

(12)

(13)

$$
\begin{aligned}
\int_{0}^{1}\left|t-\frac{\lambda}{2} \frac{b-a}{x-a}\right|^{p} d t & =\int_{0}^{\frac{\lambda}{2} \frac{b-a}{x-a}}\left(\frac{\lambda}{2} \frac{b-a}{x-a}-t\right)^{p} d t+\int_{\frac{\lambda}{2} \frac{b-a}{x-a}}^{1}\left(t-\frac{\lambda}{2} \frac{b-a}{x-a}\right)^{p} d t \\
& =\frac{1}{p+1}\left[\left(\frac{\lambda}{2} \frac{b-a}{x-a}\right)^{p+1}+\left(1-\frac{\lambda}{2} \frac{b-a}{x-a}\right)^{p+1}\right]
\end{aligned}
$$

$$
\begin{aligned}
\int_{0}^{1}\left|t-\frac{\lambda}{2} \frac{b-a}{b-x}\right|^{p} d t & =\int_{0}^{\frac{\lambda}{2} \frac{b-a}{b-x}}\left(\frac{\lambda}{2} \frac{b-a}{b-x}-t\right)^{p} d t+\int_{\frac{\lambda}{2} \frac{b-a}{b-x}}^{1}\left(t-\frac{\lambda}{2} \frac{b-a}{b-x}\right)^{p} d t \\
& =\frac{1}{p+1}\left[\left(\frac{\lambda}{2} \frac{b-a}{b-x}\right)^{p+1}+\left(1-\frac{\lambda}{2} \frac{b-a}{b-x}\right)^{p+1}\right], \\
\int_{0}^{1}\left|f^{\prime}(t x+(1-t) a)\right|^{q} d t & \leq \int_{0}^{1}\left[t^{s}\left|f^{\prime}(x)\right|^{q}+(1-t)^{s}\left|f^{\prime}(a)\right|^{q}\right] d t \\
& =\frac{\left|f^{\prime}(x)\right|^{q}+\left|f^{\prime}(a)\right|^{q}}{s+1} \leq \frac{2 M^{q}}{s+1}
\end{aligned}
$$

and

$$
\begin{aligned}
\int_{0}^{1}\left|f^{\prime}(t x+(1-t) b)\right|^{q} d t & \leq \int_{0}^{1}\left[t^{s}\left|f^{\prime}(x)\right|^{q}+(1-t)^{s}\left|f^{\prime}(b)\right|^{q}\right] d t \\
& =\frac{\left|f^{\prime}(x)\right|^{q}+\left|f^{\prime}(b)\right|^{q}}{s+1} \leq \frac{2 M^{q}}{s+1} .
\end{aligned}
$$

The proof is thus completed.

Corollary 1. Let the assumptions of Theorem 3 hold. Then for any $\lambda \in[0,1]$ we have

$$
\begin{aligned}
& \left|(1-\lambda) f\left(\frac{a+b}{2}\right)+\lambda \frac{f(a)+f(b)}{2}-\frac{1}{b-a} \int_{a}^{b} f(t) d t\right| \\
\leq & {\left[\frac{\lambda^{p+1}+(1-\lambda)^{p+1}}{p+1}\right]^{\frac{1}{p}}\left(\frac{2}{s+1}\right)^{\frac{1}{q}} \frac{M(b-a)}{2} . }
\end{aligned}
$$

Proof. We set $x=\frac{a+b}{2}$ in (11) to get (14).

Remark 3 . If we take $\lambda=0$ and $\lambda=1$ in (14), we get a midpoint type inequality

$$
\left|f\left(\frac{a+b}{2}\right)-\frac{1}{b-a} \int_{a}^{b} f(t) d t\right| \leq \frac{M(b-a)}{2(1+p)^{\frac{1}{p}}}\left(\frac{2}{s+1}\right)^{\frac{1}{q}}
$$


and a trapezoid type inequality

$$
\left|\frac{f(a)+f(b)}{2}-\frac{1}{b-a} \int_{a}^{b} f(t) d t\right| \leq \frac{M(b-a)}{2(1+p)^{\frac{1}{p}}}\left(\frac{2}{s+1}\right)^{\frac{1}{q}}
$$

which recapture (2) for $s=1$.

If we take $\lambda=\frac{1}{3}$ in (14), we get a Simpson type inequality

$$
\begin{aligned}
& \quad\left|\frac{1}{6}\left[f(a)+4 f\left(\frac{a+b}{2}\right)+f(b)\right]-\frac{1}{b-a} \int_{a}^{b} f(t) d t\right| \\
& \leq \frac{M(b-a)}{2}\left(\frac{1+2^{p+1}}{3^{p+1}(p+1)}\right)^{\frac{1}{p}}\left(\frac{2}{s+1}\right)^{\frac{1}{q}},
\end{aligned}
$$

and if we take $\theta=\frac{1}{2}$ in (14), we get an averaged midpoint-trapezoid type inequality as

(15)

$$
\left|\frac{1}{4}\left[f(a)+2 f\left(\frac{a+b}{2}\right)+f(b)\right]-\frac{1}{b-a} \int_{a}^{b} f(t) d t\right| \leq \frac{M(b-a)}{4(1+p)^{\frac{1}{p}}}\left(\frac{2}{s+1}\right)^{\frac{1}{q}} .
$$

Remark 4. If we take $\lambda=0$ in (11), we recapture the inequality (7) and thus Theorem 5 may be regarded as a generalization of Theorem 3 , and, it is interesting to notice that the smallest bound for (11) is obtained at $x=\frac{a+b}{2}$ and $\lambda=\frac{1}{2}$. Thus the averaged midpoint-trapezoid inequality (15) is optimal in the current situation.

Theorem 6. Let the assumptions of Theorem 4 hold. Then for all $\lambda \in[0,1]$ and $x \in\left[a+\lambda \frac{b-a}{2}, b-\lambda \frac{b-a}{2}\right]$, the following inequality holds:

$$
\begin{aligned}
& \left|(1-\lambda) f(x)+\lambda \frac{f(a)+f(b)}{2}-\frac{1}{b-a} \int_{a}^{b} f(t) d t\right| \\
& \leq \frac{2^{\frac{2}{q}-1} M}{[(s+1)(s+2)]^{\frac{1}{q}}}\left\{\frac{(x-a)^{2}}{b-a}\left[\left(\frac{\lambda}{2} \frac{b-a}{x-a}\right)^{2}+\left(1-\frac{\lambda}{2} \frac{b-a}{x-a}\right)^{2}\right]^{1-\frac{1}{q}}\right. \\
& \quad \times\left[\left(\frac{\lambda}{2} \frac{b-a}{x-a}\right)^{s+2}+\left(1-\frac{\lambda}{2} \frac{b-a}{x-a}\right)^{s+2}+\frac{s}{2}\right]^{\frac{1}{q}} \\
& \quad+\frac{(b-x)^{2}}{b-a}\left[\left(\frac{\lambda}{2} \frac{b-a}{b-x}\right)^{2}+\left(1-\frac{\lambda}{2} \frac{b-a}{b-x}\right)^{2}\right]^{1-\frac{1}{q}} \\
& \left.\quad \times\left[\left(\frac{\lambda}{2} \frac{b-a}{b-x}\right)^{s+2}+\left(1-\frac{\lambda}{2} \frac{b-a}{b-x}\right)^{s+2}+\frac{s}{2}\right]^{\frac{1}{q}}\right\} .
\end{aligned}
$$

Proof. Using Lemma 1 and the Hölder inequality, we have

$$
\left|(1-\lambda) f(x)+\lambda \frac{f(a)+f(b)}{2}-\frac{1}{b-a} \int_{a}^{b} f(t) d t\right|
$$




$$
\begin{aligned}
& \leq \frac{(x-a)^{2}}{b-a} \int_{0}^{1}\left|t-\frac{\lambda}{2} \frac{b-a}{x-a} \| f^{\prime}(t x+(1-t) a)\right| d t \\
& +\frac{(b-x)^{2}}{b-a} \int_{0}^{1}\left|t-\frac{\lambda}{2} \frac{b-a}{b-x}\right|\left|f^{\prime}(t x+(1-t) b)\right| d t \\
& =\frac{(x-a)^{2}}{b-a} \int_{0}^{1}\left|t-\frac{\lambda}{2} \frac{b-a}{x-a}\right|^{1-\frac{1}{q}}\left|t-\frac{\lambda}{2} \frac{b-a}{x-a}\right|^{\frac{1}{q}}\left|f^{\prime}(t x+(1-t) a)\right| d t \\
& +\frac{(b-x)^{2}}{b-a} \int_{0}^{1}\left|t-\frac{\lambda}{2} \frac{b-a}{b-x}\right|^{1-\frac{1}{q}}\left|t-\frac{\lambda}{2} \frac{b-a}{b-x}\right|^{\frac{1}{q}}\left|f^{\prime}(t x+(1-t) b)\right| d t \\
& \leq \frac{(x-a)^{2}}{b-a}\left(\int_{0}^{1}\left|t-\frac{\lambda}{2} \frac{b-a}{x-a}\right| d t\right)^{1-\frac{1}{q}} \\
& \times\left(\int_{0}^{1}\left|t-\frac{\lambda}{2} \frac{b-a}{x-a}\right|\left|f^{\prime}(t x+(1-t) a)\right|^{q} d t\right)^{\frac{1}{q}} \\
& +\frac{(b-x)^{2}}{b-a}\left(\int_{0}^{1}\left|t-\frac{\lambda}{2} \frac{b-a}{b-x}\right| d t\right)^{1-\frac{1}{q}} \\
& \times\left(\int_{0}^{1}\left|t-\frac{\lambda}{2} \frac{b-a}{b-x}\right|\left|f^{\prime}(t x+(1-t) b)\right|^{q} d t\right)^{\frac{1}{q}} \\
& \leq \frac{2^{\frac{2}{q}-1} M}{[(s+1)(s+2)]^{\frac{1}{q}}}\left\{\frac{(x-a)^{2}}{b-a}\left[\left(\frac{\lambda}{2} \frac{b-a}{x-a}\right)^{2}+\left(1-\frac{\lambda}{2} \frac{b-a}{x-a}\right)^{2}\right]^{1-\frac{1}{q}}\right. \\
& \times\left[\left(\frac{\lambda}{2} \frac{b-a}{x-a}\right)^{s+2}+\left(1-\frac{\lambda}{2} \frac{b-a}{x-a}\right)^{s+2}+\frac{s}{2}\right]^{\frac{1}{q}} \\
& +\frac{(b-x)^{2}}{b-a}\left[\left(\frac{\lambda}{2} \frac{b-a}{b-x}\right)^{2}+\left(1-\frac{\lambda}{2} \frac{b-a}{b-x}\right)^{2}\right]^{1-\frac{1}{q}} \\
& \left.\left[\left(\frac{\lambda}{2} \frac{b-a}{b-x}\right)^{s+2}+\left(1-\frac{\lambda}{2} \frac{b-a}{b-x}\right)^{s+2}+\frac{s}{2}\right]^{\frac{1}{q}}\right\},
\end{aligned}
$$

since

$$
\int_{0}^{1}\left|t-\frac{\lambda}{2} \frac{b-a}{x-a}\right| d t=\frac{1}{2}\left[\left(\frac{\lambda}{2} \frac{b-a}{x-a}\right)^{2}+\left(1-\frac{\lambda}{2} \frac{b-a}{x-a}\right)^{2}\right]
$$

in case $p=1$ of (12) and

$$
\int_{0}^{1}\left|t-\frac{\lambda}{2} \frac{b-a}{x-a}\right| d t=\frac{1}{2}\left[\left(\frac{\lambda}{2} \frac{b-a}{x-a}\right)^{2}+\left(1-\frac{\lambda}{2} \frac{b-a}{x-a}\right)^{2}\right]
$$

in case $p=1$ of (13),

$$
\begin{aligned}
& \int_{0}^{1}\left|t-\frac{\lambda}{2} \frac{b-a}{x-a}\right|\left|f^{\prime}(t x+(1-t) a)\right|^{q} d t \\
= & \int_{0}^{\frac{\lambda}{2} \frac{b-a}{x-a}}\left(\frac{\lambda}{2} \frac{b-a}{x-a}-t\right)\left|f^{\prime}(t x+(1-t) a)\right|^{q} d t
\end{aligned}
$$




$$
\begin{aligned}
& \left.+\int_{\frac{\lambda}{2} \frac{b-a}{x-a}}^{1}\left(t-\frac{\lambda}{2} \frac{b-a}{x-a}\right)\left|f^{\prime}(t x+(1-t) a)\right|^{q} d t\right] \\
\leq & \int_{0}^{\frac{\lambda}{2} \frac{b-a}{x-a}}\left(\frac{\lambda}{2} \frac{b-a}{x-a}-t\right)\left[t^{s}\left|f^{\prime}(x)\right|^{q}+(1-t)^{s}\left|f^{\prime}(a)\right|^{q}\right] d t \\
& \left.+\int_{\frac{\lambda}{2} \frac{b-a}{x-a}}^{1}\left(t-\frac{\lambda}{2} \frac{b-a}{x-a}\right)\left[t^{s}\left|f^{\prime}(x)\right|^{q}+(1-t)^{s}\left|f^{\prime}(a)\right|^{q}\right] d t\right] \\
= & {\left[\frac{1}{s+2}-\frac{\lambda}{2} \frac{b-a}{x-a} \frac{1}{s+1}+\frac{2}{(s+1)(s+2)}\left(\frac{\lambda}{2} \frac{b-a}{x-a}\right)^{s+2}\right]\left|f^{\prime}(x)\right|^{q} } \\
& +\left[\frac{1}{s+2}-\left(1-\frac{\lambda}{2} \frac{b-a}{x-a}\right) \frac{1}{s+1}+\frac{2}{(s+1)(s+2)}\left(1-\frac{\lambda}{2} \frac{b-a}{x-a}\right)^{s+2}\right]\left|f^{\prime}(a)\right|^{q} \\
\leq & \frac{2 M^{q}}{(s+1)(s+2)}\left[\left(\frac{\lambda}{2} \frac{b-a}{x-a}\right)^{s+2}+\left(1-\frac{\lambda}{2} \frac{b-a}{x-a}\right)^{s+2}+\frac{s}{2}\right]
\end{aligned}
$$

and

$$
\begin{aligned}
& \int_{0}^{1}\left|t-\frac{\lambda}{2} \frac{b-a}{b-x}\right|\left|f^{\prime}(t x+(1-t) b)\right|^{q} d t \\
= & \int_{0}^{\frac{\lambda}{2} \frac{b-a}{b-x}}\left(\frac{\lambda}{2} \frac{b-a}{b-x}-t\right)\left|f^{\prime}(t x+(1-t) b)\right|^{q} d t \\
& \left.+\int_{\frac{\lambda}{2} \frac{b-a}{b-x}}^{1}\left(t-\frac{\lambda}{2} \frac{b-a}{b-x}\right)\left|f^{\prime}(t x+(1-t) b)\right|^{q} d t\right] \\
\leq & \int_{0}^{\frac{\lambda}{2} \frac{b-a}{b-x}}\left(\frac{\lambda}{2} \frac{b-a}{b-x}-t\right)\left[t^{s}\left|f^{\prime}(x)\right|^{q}+(1-t)^{s}\left|f^{\prime}(b)\right|^{q}\right] d t \\
& \left.+\int_{\frac{\lambda}{2} \frac{b-a}{b-x}}^{1}\left(t-\frac{\lambda}{2} \frac{b-a}{b-x}\right)\left[t^{s}\left|f^{\prime}(x)\right|^{q}+(1-t)^{s}\left|f^{\prime}(b)\right|^{q}\right] d t\right] \\
= & {\left[\frac{1}{s+2}-\frac{\lambda}{2} \frac{b-a}{b-x} \frac{1}{s+1}+\frac{2}{(s+1)(s+2)}\left(\frac{\lambda}{2} \frac{b-a}{b-x}\right)^{s+2}\right]\left|f^{\prime}(x)\right|^{q} } \\
& +\left[\frac{1}{s+2}-\left(1-\frac{\lambda}{2} \frac{b-a}{b-x}\right) \frac{1}{s+1}+\frac{2}{(s+1)(s+2)}\left(1-\frac{\lambda}{2} \frac{b-a}{b-x}\right)^{s+2}\right]\left|f^{\prime}(b)\right|^{q} \\
\leq & \frac{2 M^{q}}{(s+1)(s+2)}\left[\left(\frac{\lambda}{2} \frac{b-a}{b-x}\right)^{s+2}+\left(1-\frac{\lambda}{2} \frac{b-a}{b-x}\right)^{s+2}+\frac{s}{2}\right] .
\end{aligned}
$$

The proof is thus completed.

Corollary 2. Let the assumptions of Theorem 4 hold. Then for any $\lambda \in[0,1]$ we have

$$
\left|(1-\lambda) f\left(\frac{a+b}{2}\right)+\lambda \frac{f(a)+f(b)}{2}-\frac{1}{b-a} \int_{a}^{b} f(t) d t\right|
$$




$$
\leq\left[\frac{\lambda^{2}+(1-\lambda)^{2}}{2}\right]^{1-\frac{1}{q}}\left[\frac{s+2 \lambda^{s+2}+2(1-\lambda)^{s+2}}{(s+1)(s+2)}\right]^{\frac{1}{q}} \frac{M(b-a)}{2} .
$$

Proof. We set $x=\frac{a+b}{2}$ in (16) to get (17).

Remark 5. If we take $\lambda=0$ and $\lambda=1$ in (17), we get a midpoint type inequality

$$
\left|f\left(\frac{a+b}{2}\right)-\frac{1}{b-a} \int_{a}^{b} f(t) d t\right| \leq \frac{M(b-a)}{4}\left(\frac{2}{s+1}\right)^{\frac{1}{q}}
$$

and a trapezoid type inequality

$$
\left|\frac{f(a)+f(b)}{2}-\frac{1}{b-a} \int_{a}^{b} f(t) d t\right| \leq \frac{M(b-a)}{4}\left(\frac{2}{s+1}\right)^{\frac{1}{q}},
$$

which recapture (5) and (6) for $s=1$, respectively.

If we take $\lambda=\frac{1}{3}$ in (17), we get a Simpson type inequality

$$
\begin{aligned}
& \left|\frac{1}{6}\left[f(a)+4 f\left(\frac{a+b}{2}\right)+f(b)\right]-\frac{1}{b-a} \int_{a}^{b} f(t) d t\right| \\
\leq & \frac{5 M(b-a)}{36}\left[\frac{18\left(2+2^{s+3}+3^{s+2}\right)}{5(s+1)(s+2) 3^{s+2}}\right]^{\frac{1}{q}},
\end{aligned}
$$

and if we take $\theta=\frac{1}{2}$ in (17), we get an averaged midpoint-trapezoid type inequality as

$$
\begin{aligned}
& \left|\frac{1}{4}\left[f(a)+2 f\left(\frac{a+b}{2}\right)+f(b)\right]-\frac{1}{b-a} \int_{a}^{b} f(t) d t\right| \\
\leq & \frac{M(b-a)}{8}\left[\frac{1+2^{s} s}{(s+1)(s+2) 2^{s-2}}\right]^{\frac{1}{q}} .
\end{aligned}
$$

Remark 6. If we take $\lambda=0$ in (16), we recapture the inequality (8) and thus Theorem 6 may be regarded as a generalization of Theorem 4, and, it is interesting to notice that the smallest bound for (16) is obtained at $x=\frac{a+b}{2}$ and $\lambda=\frac{1}{2}$. Thus the averaged midpoint-trapezoid inequality (18) is optimal in the current situation.

Remark 7. It should be noticed that the inequality (18) is better than the inequality (15). In fact, it is not difficult to find that $2-s-2^{1-s} \geq 0$ for $s \in(0,1]$ by differentiation, and then we can deduce that $\frac{1+2^{s} s}{(s+1)(s+2) 2^{s-2}} \leq \frac{2}{s+1}$ for $s \in(0,1]$ by elementary algebra.

\section{Applications to special means}

We now consider the applications of the averaged midpoint-trapezoid inequalities (15) and (18) to the following special means: 
(1) The arithmetic mean:

$$
A=A(a, b):=\frac{a+b}{2}, a, b \geq 0 .
$$

(2) The $p$-logarithmic mean:

$$
L_{p}=L_{p}(a, b)=\left[\frac{b^{p+1}-a^{p+1}}{(p+1)(b-a)}\right]^{\frac{1}{p}}, a \neq b, a, b>0 .
$$

From known Example 1 in [4], we may find that for any $s \in(0,1)$ and $\beta>0$, $f:[0, \infty) \rightarrow[0, \infty), f(t)=\beta t^{s}, f \in K_{s}^{2}$.

Using the averaged midpoint-trapezoid type inequalities (15) and (18), some new inequalities are derived for the above means.

Proposition 1. Let $0<a<b, p, q>1, \frac{1}{p}+\frac{1}{q}=1$ and $s \in\left(0, \frac{1}{q}\right)$. Then we have

$$
\left|\frac{1}{2} A\left(a^{s+1}, b^{s+1}\right)+\frac{1}{2} A^{s+1}(a, b)-L_{s+1}^{s+1}(a, b)\right| \leq \frac{(b-a) b^{s}}{2}\left(\frac{s+1}{2 p+2}\right)^{\frac{1}{p}} .
$$

Proof. The assertion follows from applied the inequality (15) to the function $f(t)=t^{s+1}, t \in[a, b]$ and $s \in\left(0, \frac{1}{q}\right)$, which implies that $f^{\prime}(t)=(s+1) t^{s}$, $t \in[a, b]$ and $\left|f^{\prime}(t)\right|^{q}=(s+1)^{q} t^{q s}, t \in[a, b]$ is a $s$-convex function in the second sense since $q s \in(0,1)$ and $(s+1)^{q}>0$, and we may take $M=(s+1) b^{s}$.

Proposition 2. Let $0<a<b, q \geq 1$ and $s \in\left(0, \frac{1}{q}\right)$. Then we have

$$
\begin{aligned}
& \left|\frac{1}{2} A\left(a^{s+1}, b^{s+1}\right)+\frac{1}{2} A^{s+1}(a, b)-L_{s+1}^{s+1}(a, b)\right| \\
\leq & \frac{(b-a) b^{s}(s+1)^{1-\frac{1}{q}}}{8}\left(\frac{1+2^{s} s}{(s+2) 2^{s-2}}\right)^{\frac{1}{q}} .
\end{aligned}
$$

Proof. The assertion follows from applied the inequality (18) to the function $f(t)=t^{s+1}, t \in[a, b]$ and $s \in\left(0, \frac{1}{q}\right)$, which implies that $f^{\prime}(t)=(s+1) t^{s}$, $t \in[a, b]$ and $\left|f^{\prime}(t)\right|^{q}=(s+1)^{q} t^{q s}, t \in[a, b]$ is a $s$-convex function in the second sense since $q s \in(0,1)$ and $(s+1)^{q}>0$, and we may take $M=(s+1) b^{s}$.

\section{References}

[1] M. Alomari, M. Darus, S. S. Dragomir, and P. Cerone, Ostrowski type inequalities for functions whose derivatives are s-convex in the second sense, Appl. Math. Lett. 23 (2010), no. 1, 1071-1076.

[2] S. S. Dragomir and R. P. Agarwal, Two inequalities for differentiable mappings and applications to special means of real numbers and to trapezoidal formula, Appl. Math. Lett. 11 (1998), no. 5, 91-95.

[3] S. S. Dragomir, P. Cerone, and J. Roumeliotis, A new generalization of Ostrowski integral inequality for mappings whose derivatives are bounded and applications in numerical integration and for special means, Appl. Math. Lett. 13 (2000), no. 1, 19-25.

[4] H. Hudzik and L. Maligranda, Some remarks on s-convex functions, Aequationes Math. 48 (1994), no. 1, 100-111. 
[5] U. S. Kirmaci, M. K. Bakula, M. E. Özdemir, and J. Pecarić, Hadamard-type inequalities for s-convex functions, Appl. Math. Comput. 193 (2007), no. 1, 26-35.

[6] C. E. M. Pearce and J. Pečarić, Inequalities for differentiable mappings with applications to special means and quadrature formulae, Appl. Math. Lett. 13 (2000), no. 2, 51-55.

Institute of Applied Mathematics

SCHOOL OF SCIENCE

University of Science and Technology Liaoning

Anshan 114051, Liaoning, P. R. China

E-mail address: lewzheng@163.net 